\title{
SHADOW DETECTION IMPROVEMENT USING SPECTRAL INDICES AND MORPHOLOGICAL OPERATORS IN URBAN AREAS IN HIGH RESOLUTION IMAGES
}

\author{
S. C. Azevedo ${ }^{\text {a }}$, E. A. Silva ${ }^{\text {b }}$, M. M. Pedrosa ${ }^{\text {a }}$ \\ a Postgraduate Program in Cartographic Science - Univ. Estadual Paulista (UNESP) - 19060-900 - Presidente Prudente - SP, Brazil \\ -samara_calcado@hotmail.com; miriammmp@ hotmail.com \\ ${ }^{\mathrm{b}}$ Department of Cartography - UNESP - Presidente Prudente - SP, Brazil - silva.erivaldo@ gmail.com
}

KEY WORDS: Shadow detection, high resolution, remote sensing, urban areas.

\begin{abstract}
:
While high-resolution remote sensing images have increased application possibilities for urban studies, the large number of shadow areas has created challenges to processing and extracting information from these images. Furthermore, shadows can reduce or omit information from the surface as well as degrading the visual quality of images. The pixels of shadows tend to have lower radiance response within the spectrum and are often confused with low reflectance targets. In this work, a shadow detection method was proposed using a morphological operator for dark pattern identification combined with spectral indices. The aims are to avoid misclassification in shadow identification through properties provided by them on color models and, therefore, to improve shadow detection accuracy. Experimental results were tested applying the panchromatic and multispectral band of WorldView-2 image from São Paulo city in Brazil, which is a complex urban environment composed by high objects like tall buildings causing large shadow areas. Black top-hat with area injunction was applied in PAN image and shadow identification performance has improved with index as Normalized Difference Vegetation Index (NDVI) and Normalized Saturation-Value Difference Index (NSDVI) ratio from HSV color space obtained from pansharpened multispectral WV-2 image. An increase in distinction between shadows and others objects was observed, which was tested for the completeness, correctness and quality measures computed, using a created manual shadow mask as reference. Therefore, this method can contribute to overcoming difficulties faced by other techniques that need shadow detection as a first necessary preprocessing step, like object recognition, image matching, 3D reconstruction, etc.
\end{abstract}

\section{INTRODUCTION}

High spatial resolution satellite images have opened a new era in extracting information from the earth's surface, particularly in urban environments, where features such as buildings, roads, vehicles and trees become detailed and easier to distinguish. On the other hand, a large surface area obscured by shadows cast by these tall objects was introduced with the increase in spatial resolution, and can reduce useful information from targets.

Although shadows can be a valuable cue to image interpretation, since it is possible to make inferences about the 3D surface and target information, more recently, shadow areas have been seen as an unwanted feature in digital image processing techniques (Arévalo et al., 2008). For example, shadows tend to be classified as part of the foreground and decrease results in tracking performance in object detection and recognition in video sequence (Sanin et al., 2012). In addition, shadows also interfere with tasks like remote sensing (RS) image classification, where significant portions of land cover remain unknown due to shadow effects.

Therefore, shadow removal is an unavoidable task to be seen as preprocessing for many applications. The first step is to identify pixels that are affected by shadows. Many algorithms have been proposed in the literature on shadow detection. Some modelbased shadow detection can project shadow location from prior knowledge about the sensor source of illumination and 3D geometry of objects in the scene. While this technique take advantage of a solved mathematical problem, there are limitations to the availability of this information. Also, models must be compatible with high-resolution images (Adeline et al., 2013). In this case, the accuracy of the shadow identification process deteriorates instead of improving.

Fortunately, shadows exhibit singular behaviour on images that can be used to separate them from other objects. In propertybased techniques, shadow detection is performed using features such as brightness and color space based on some specific spectral assumptions. Tsai (2006) compared several invariant colour spaces (HIS, HSV, HCV, YIQ, and $\mathrm{YCbCr}$ ) to detect shadows in aerial photos using spectral ratio and automatic threshold estimates from Otsu's method. Results showed good performance in segmenting shadow regions, but like most property-based methods, false detection was observed and distorted other objects in the scene. Thus, attributes like an extra feature image and variance were applied as an additional step in order to avoid misclassification (Dare, 2005; Cai et al., 2010).

Regarding the importance of shadow identification, in this paper we present an alternative semi-automatic shadow detection methodology based on shadow properties by integrating morphological image processing (MIP) and spectral information. We aim to verify, through quality measure rates, which spectral index combination improves a proposed shadow detection algorithm, in order to minimize misclassification and enhance shadow identification reliability.

\footnotetext{
* Corresponding author. This is useful to know for communication with the appropriate person in cases with more than one author.
} 


\section{SHADOW DETECTION APPROACH}

High-resolution urban RS images are covered by many shadow regions, especially in dense and complex urban environments. In the case of passive sensors, shadows occur when a fraction of direct light from a source of illumination is blocked (Arévalo et al., 2008). Thus, responses arriving at the sensor from shadow regions tend to have lower sensor radiance across the whole spectrum than their lighted neighbouring areas (Adeline et al., 2013). Based on this principal shadow property, MIP is an alternative to detect shadow, since it acts on minimum and maximum values, in the case of grey scale images like the RS images. We therefore used operators applied to valley detection, due to the fact that valleys on topographic surfaces have smaller values, as explained in the following.

\subsection{Shadow candidates segmentation by MIP}

The concept of point-wise maximum and minimum operators (see more details in Soille, 2004) applied by MIP provides tools for detectiing dark structures from images. The principal characteristic of MIP is the use of a set of known structuring elements (SE), usually chosen according to some a priori knowledge about the geometry of the relevant structures. In shadow detection, we take advantage of adaptive properties from area closing morphological operator, since, in this operation, the SE adapts to the geometric structure based on the area injunction defined in accordance with the size of structures to be filtered (Soille, 2004).

An important issue in this case is that shadow areas take on distinct shapes due to the different objects that cast them. In addition, MIP is capable of modifying the image while, at the same time, preserving geometric structures of interest through arithmetic differences between images, called top-hats. Shadow segmentation can be achieve using filtering by the black top-hat with area injunction (Equation 1), where $\emptyset_{\lambda}(f)$ is the area closing of image $f$ (Soille, 2004).

$$
\mathrm{BTH}(f)=\phi_{\lambda}(f)-f
$$

So, $\emptyset_{\lambda}(f)$ removes all dark components in the image that do not fit in the connected Structuring Elements (SE), whose size in number of pixels equals the area parameter $\lambda$. The size of $\lambda$ was empirically set and varies with spatial image resolution. Thus, interesting features are eliminated and recovered when arithmetic difference with the original image is performed. Finally, shadows are detected by automatic binarization with the Otsu (1979) method.

Nonetheless, Rayleigh scattering contributes with shadow response. This can cause false detection and confusion between targets that have low response patterns. Fortunately, shadows have others properties for color images that can be exploited as a means of improving shadow detection accuracy. Also, other targets that may cause confusions must be detected using spectral information and elimination from final shadow detection results.

\subsection{Shadow Spectral Segmentation}

Standard color space such as RGB, contains both radiance and chromaticity information in each channel (Adeline et al., 2013). Besides having low radiance, shadow presents other properties that have been studied in the literature. Polidorio et al. (2003) observed that shadows have low luminance, because they do not receive a direct flow of luminous energy, and have high saturation on violet and blue wavelengths due to the Rayleigh scattering effect. Shaded areas were segmented by thresholding the results from difference images between the saturation and the intensity information on the normalized hue, saturation, and intensity (HSI) color space.

Methods that use color features for detecting shadows generally choose a colour space that provides better separation between chromacity and intensity than the RGB colour space. The hue, saturation, and value (HSV) color space also highlights the presence of shadows. In the case of the HSV color model, shadows tend to have high saturation (Fig. 1c) and low value (Fig. 1d). A normalized saturation-value difference index (NSVDI), can be constructed by the Equation 2 and applied to identify shadows (Ma et al., 2008).

$$
\text { NSDVI }=\frac{S-V}{S+V}
$$

After calculating the NSDVI, a thresholding process is applied, once shadows tend to give the positive values, more than zero on NSDVI matrix results. Nonetheless, misclassified and false detection errors also can occur for shadow invariant methods. This is because, in some case, targets can present similar behaviour in HSV color space. For example, vegetation areas similarly have high saturation (Fig. 1c) and low value (Fig. 1d), which can be included on NSDVI results.

Fortunately, vegetation exhibits well known opposite behaviour used to highlight these areas in the near-infrared (NIR) and red (R) bands. The Normalized Difference Vegetation Index (NDVI) defined by Equation 3 (Wang et al., 2005) is one of the most widely applied vegetation indices.

$$
N D V I=\frac{N I R-R}{N I R+R}
$$

The NDVI result matrix can be easily thresholded to separate vegetation from other objects since these areas have higher values than the rest of the image. Consequently, combination with NSDVI allows only the real shadows to be detected, which can reflect to improve shadow detection accuracy.

\subsection{Shadow Detection Assessment}

Evaluation results of extraction/detection procedures are necessary, especially in automatic or semi-automatic methods, so users can check the quality of data obtained based on values or statistical metrics that allow validation of the process

Completeness, correctness and quality metrics were adopted in this work to verify which approach was best able to improve shadow detection and to avoid misclassification,. The process consists of comparing the detected result with a reference shadow mask, also called ground-truth (GT), pixel by pixel. The quality measures proposed by Wiedemann et al. (1998) are described in the following.

\subsubsection{Completeness}

The first quality metric, called completeness (Com), is defined by Equation 4 and represents the percentage of shadow pixels in the GT image that have been properly detected by the approach. 
The value can vary in the interval [0:1], where the value 1 is considered ideal and shows how complete the approach is.

$$
C o m=\frac{T P}{T P+F N}
$$

TP being the true positives, i.e., the number of pixels correctly detected as shadow, and FN being the false negatives, which are shadow pixels not detected as such, in accordance with GT.

\subsubsection{Correctness}

The correctness (Cor) metric, shown in Equation 5, represents the percentage of shadow pixels correctly identified by the method, in accordance with GT, being 1 the ideal value.

$$
C o r=\frac{T P}{T P+F P}
$$

FP being the false positives, which are pixels falsely detected by the approach as shadow, but which, in reality, are not shadows.

\subsubsection{Quality}

The last metric is the quality (Qua), shown by Equation 6, and combines both previous measures, showing how good the approach is, 1 being the ideal value to be achieved.

$$
Q u a=\frac{T P}{T P+F P+F N}
$$

where the value can describe only, the quality obtained by an approach.

\section{METHODOLOGY}

The methodology aiming to detect shadow and improve results with spectral information was performed in high-resolution WV-2 image dated May 2012. The study area cover a portion of São Paulo city characterized as a complex urban area with one of the highest urban agglomeration rates in South America. Panchromatic (PAN) and 4 Multispectral (MS) bands with around $0.5 \mathrm{~m}$ and $2 \mathrm{~m}$ of spatial resolution respectively were applied, where the multispectral bands correspond to traditional visible (red, green and blue) and near-infrared NIR.

The principal component (PC) spectral sharpening method was used to create new 4 bands with improved spatial resolution compatible with PAN to carry out the method. In this fusion method, the first PC band (PC1) is replaced by a PAN band, which is first stretched to have the same mean and variance as PC1. The inverse PCA transform is then performed to obtain a new high-resolution multispectral image (Wang et al., 2005) without distortions on spectral information, as shown in Fig. 2a.

Experiments were performed with five subimages (PAN and MS) with sizes of $300 \times 300$ pixels from the WV-2 image scene. The evaluation of spectral information contribution on shadow detection was performed in three approaches illustrated in Fig. 1. The first approach consisted of segment shadow in PAN band using the black top-hat with area injunction method aforementioned. The size parameter was selected empirically, and, due to higher WV-2 resolution, this size has to be large to extract the shadow areas connected. As all images have the same resolution, only one parameter was required. In addition, the need for preprocessing was discarded, as the morphological operator top-hat applied behaves as a filter of the image structures (Azevedo et al., 2013).

In the second approach, spectral information extracted from the MS pansharpening image was used to calculate NDVI. According to the ratio acquired from NDVI, the behavior of vegetation pixels was investigated and a thresholding was easily determined to create the vegetation mask. The results were subtracted from the first approach to eliminate false shadow detection and improve results from the accuracy indices calculated.

Finally, the third approach combined the spectral indices NDVI and NSDVI calculated from the first approach to verify improvement on shadow detection from shadow property acquired in HSV invariant space color. Then, conversion of the RGB color model to HSV was carried out and results of detection rates were compared.

The results analysis consisted of comparing quality measures described in Wiedemann (1998) from the results of the three approaches. Thus, a reference shadow mask, also called groundtruth (GT) was obtained manually from these five images by selecting shadowed areas in the PAN band. The implementation of the semi-automatic methodology was carried out through MATLAB software which automatically compares the processed images with their respectively GT, calculating the detection rates between them.

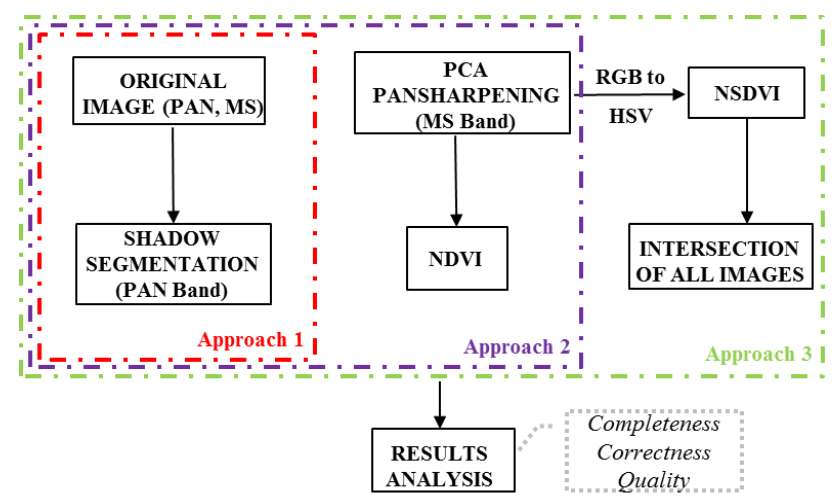

Figure 1. Diagram of approaches developed in the methodology.

\section{EXPERIMENTAL RESULTS AND DISCUSSION}

Experiments using the three approaches aforementioned were carried out with the five subimages. The PAN band and pansharpened MS data from one of the subimages, Image 1, are shown on Fig. 2 (a) and (b), respectively. PCA fusion technique was applied to provide spatial improvement with the same PAN band spatial resolution and was able to preserve spectral characteristics, as we can see on the false color composite for the pansharpened WV-2. Furthermore, there were no indications of distortion by using the PC pansharpened method, and both data could be applied together in the study. Results of the normalized saturation and value component from image 1, are shown on Fig. 2 (c) and (d), respectively.

Fig. 3 shows binary results from the approaches. It can be seen from visual analysis that the first approach (Fig. 3a) detected 
most shadow areas present, compared with original or false color composition of image 1 already shown in Fig 2, for example. We can also observe that a great part of the vegetation areas detected on NDVI mask results (Fig. 3b), did not appear on the result of the first approach. On the other hand, the binary result of NSDVI image (Fig. 3c) showed confusion with vegetation. This was expected since, as previously discussed, they have similar behaviour in S and V components (see Fig. 2(c) and (d)).

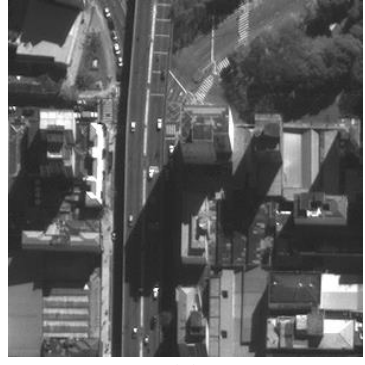

(a)

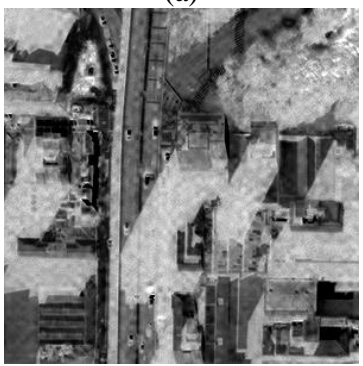

(c)

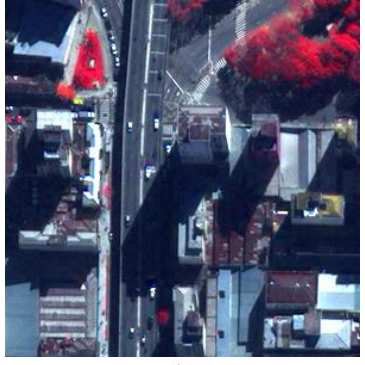

(b)

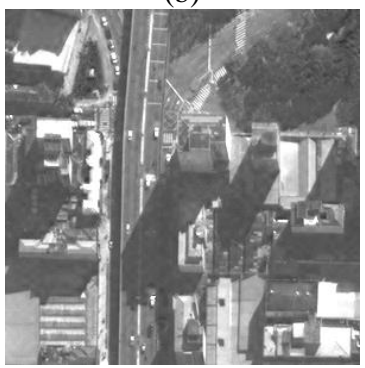

(d)
Figure 2. Examples of input image 1. (a)WV-2 Pan; (b) Pansharpened WV-2; (c) Component of S; (d)Component of V.

Table 1 summarizes measurements computed for the set of images in order to compare the performance approaches. Considering the Quality measure, which is a combination of completeness and correctness, good results were achieved. The first approach should be highlighted, because it has shown the possibility of shadowing segmentation with morphological operators by itself, in terms of all measure values.

Although, Fig. 3 (f) shows some vegetation areas at the top of the image that appear in red, which were incorrectly detected as shadow in the first approach. The performances obtained with the second approach did not present quality rates different from the previous one, probably because the first approach was able to deal with mostly vegetation areas.

Improvements were observed when subsequent approaches were applied, mainly with the third approach. Although average quality percentage has not shown expressive growth, an important role of this procedure was to diminish FP values, which is directly observed with the increasing of correctness. All tested images achieved better correctness rates with the third approach, for example, image 1 climbed from $90.7 \%$ to $98.9 \%$.

Fig. 4 shows comparison results for the others four images. Red areas representing FP, i.e., pixels incorrectly detected as shadow in the first approach. The pixels correctly identified as shadow are in green, i.e. TP, whereas the areas that have not been identified by these methods (FN) are in blue in accordance with the GT. As we can see, FP was reduced by the NSDVI spectral index, proved by improved correctness values. The sparse amount of blue areas shows that the method was adequate for detecting most shadow areas from urban images. In addition, a few small shadow areas were not labelled by GT, for example, some shadows cast by vehicles at the bottom of the third image. These areas did not achieve the effectiveness of the method. However, they contribute to FP presence.
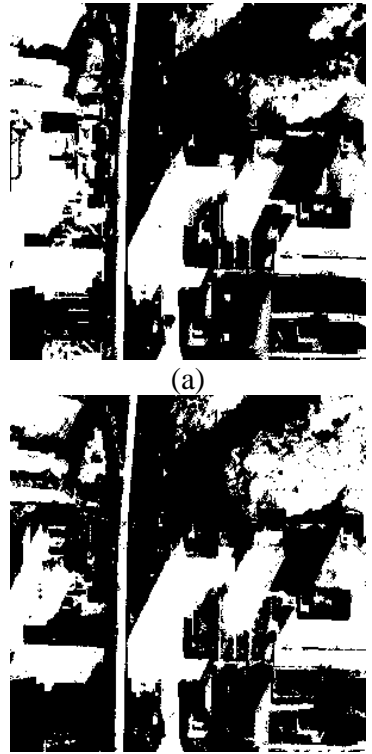

(c)

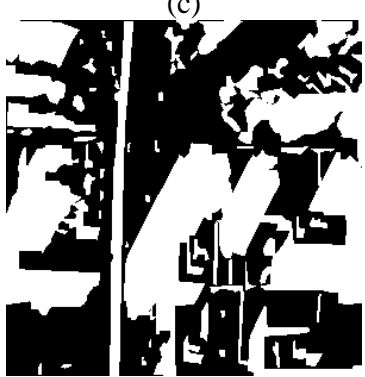

(e)
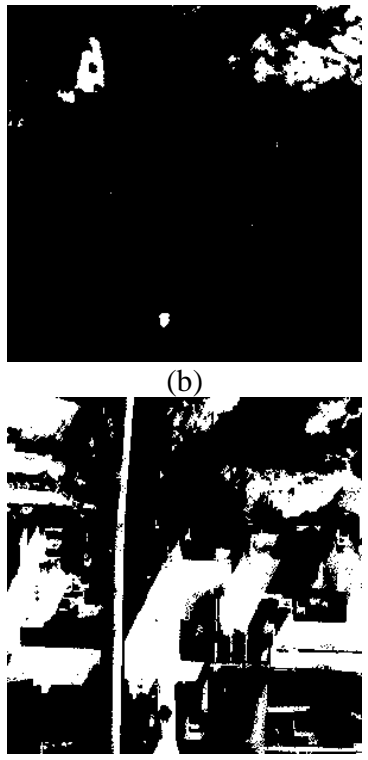

(d)

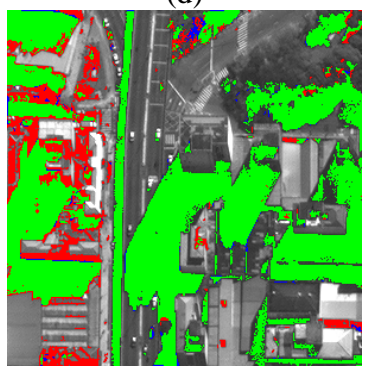

(f)
Fig. 3. Image 1 binary results for comparison. (a) First approach result; (b) NDVI mask; (c) NSDVI results; (d) Third approach result; (e) GT; (f) Comparison between (a, d, e).

\begin{tabular}{|c|c|c|c|c|c|c|c|c|c|}
\hline \multirow[b]{2}{*}{$\begin{array}{c}\text { Tested } \\
\text { Images }\end{array}$} & \multicolumn{3}{|c|}{ Approach 1} & \multicolumn{3}{|c|}{ Approach 2} & \multicolumn{3}{|c|}{ Approach 3} \\
\hline & $\begin{array}{l}\mathbf{C} \\
\mathbf{o} \\
\mathbf{m} \\
\end{array}$ & $\begin{array}{l}\mathbf{C} \\
\mathbf{o} \\
\mathbf{r} \\
\end{array}$ & $\begin{array}{l}\mathbf{Q} \\
\mathbf{u} \\
\mathbf{a}\end{array}$ & $\begin{array}{l}\mathbf{C} \\
\mathbf{o} \\
\mathbf{m} \\
\end{array}$ & $\begin{array}{l}\mathbf{C} \\
\mathbf{o} \\
\mathbf{r} \\
\end{array}$ & $\begin{array}{l}\mathbf{Q} \\
\mathbf{u} \\
\mathbf{a}\end{array}$ & $\begin{array}{l}\mathbf{C} \\
\mathbf{o} \\
\mathbf{m} \\
\end{array}$ & $\begin{array}{l}\mathbf{C} \\
\mathbf{o} \\
\mathbf{r} \\
\end{array}$ & $\begin{array}{l}\mathbf{Q} \\
\mathbf{u} \\
\mathbf{a}\end{array}$ \\
\hline $\begin{array}{c}\text { Image } \\
1\end{array}$ & 0.902 & 0.907 & 0.826 & 0.897 & 0.911 & 0.825 & 0.888 & 0.989 & 0.880 \\
\hline $\begin{array}{c}\text { Image } \\
2 \\
\end{array}$ & 0.952 & 0.916 & 0.876 & 0.939 & 0.916 & 0.865 & 0.910 & 0.982 & 0.896 \\
\hline $\begin{array}{c}\text { Image } \\
\mathbf{3} \\
\end{array}$ & 0.990 & 0.832 & 0.825 & 0.987 & 0.836 & 0.827 & 0.967 & 0.915 & 0.888 \\
\hline $\begin{array}{c}\text { Image } \\
4 \\
\end{array}$ & 0.989 & 0.964 & 0.955 & 0.988 & 0.965 & 0.954 & 0.973 & 0.980 & 0.954 \\
\hline $\begin{array}{c}\text { Image } \\
5 \\
\end{array}$ & 0.985 & 0.937 & 0.924 & 0.977 & 0.942 & 0.922 & 0.960 & 0.968 & 0.931 \\
\hline Mean & 0.963 & 0.911 & 0.881 & 0.957 & 0.914 & 0.878 & 0.939 & 0.966 & 0.896 \\
\hline
\end{tabular}

Table 1. Comparison of Completeness (Com), Correctness (Cor) and Quality (Qua) index for the three approaches. 

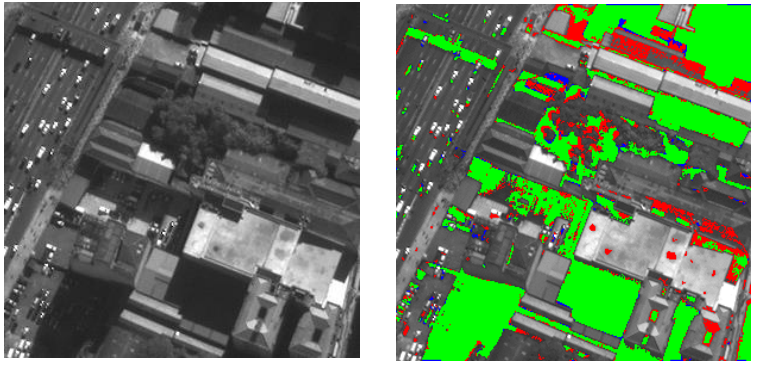

Image 2
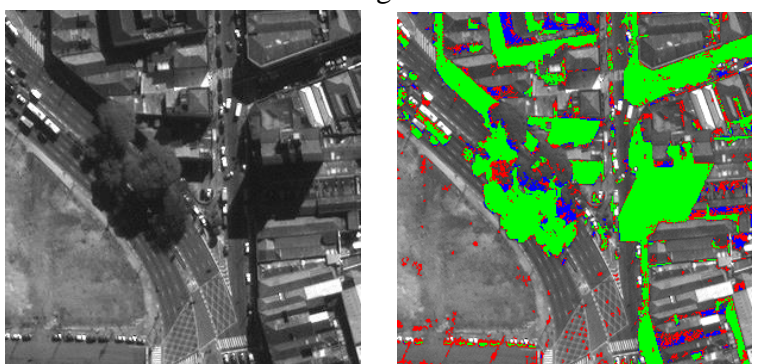

Image 3
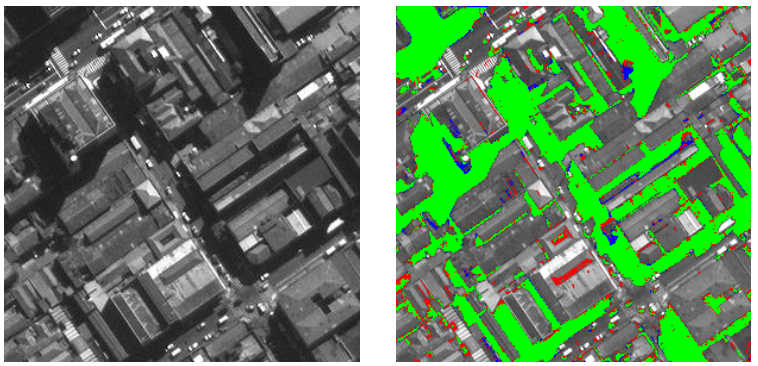

Image 4
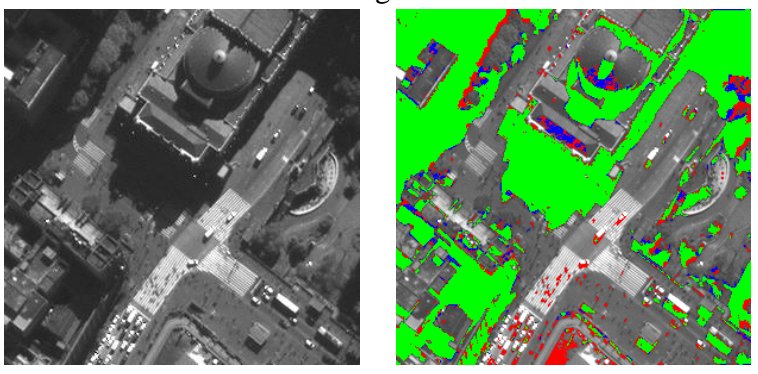

Image 5

Figure 4. Comparison results for Image 2, 3, 4 and 5, respectively. (a) Original image; (b) Compared output results.

\section{CONCLUSIONS}

This paper presented a semi-automatic shadow detection methodology aimed at enhancing performance index through the properties provided for shadow in multispectral images. The first approach applied MIP to identify areas of interest in the WV-2 panchromatic band from São Paulo city images. The results from the five images tested showed that the method succeeded in identifying most shadows areas, which was proved by completeness rates. This demonstrated property-based shadow detection using black top-hat, taking advantage of contextual information provided MIP to segment the image into shadow and nonshadow regions.

The advantage of this approach lies in the fact that only the input image was necessary to carry out the method. However, an improvement was noted when spectral information was available. Thus, from pansharpening four WV-2 MS bands (visible and NIR), the NDVI and NSDVI ratio map could be obtained and combined to enhance shadow discrimination, once they present more properties on other color model spaces.

The evaluation of the approaches was performed through completeness, correctness and quality rates. Results showed that, even though a significant increase in mean quality percentage rate was not observed, the decrease of FP pixels erroneously detected as shadows in the first method resulted in an increase in the reliability of the method through higher correctness rates observed after applying combined spectral indices. The methodology must be tested with aerial imagery and we also intend to automate the method with the use of a single area parameter for any spatial resolution input image.

\section{ACKNOWLEDGEMENTS}

The authors would like to thank CAPES for support and FAPESP (Proc. $n^{\circ}$ 2013/25257-4) for financial support given to the development of this work, and to the project for providing a research grant.

\section{REFERENCES}

Adeline, K.R.M., Chen, M., Briottet, X., Pang, S.K. and Paparoditis, N., 2013. Shadow detection in very high spatial resolution aerial images: a comparative study. ISPRS Journal of Photogrammetry and Remote Sensing, 80, pp. 21-38.

Arévalo, V., González, J. and Ambrosio, G., 2008. Shadow detection in color high- resolution satellite images. International Journal of Remote Sensing, 29 (7), pp. 19451963.

Azevedo, S. C., Pagamisse, A., Silva, E. A. and Cardim, G. P. 2013. Study of the Preprocessing Influence in the Accuracy of Semi-Automated Shadow Detection Approach. Journal of Communication and Computer, v. 10, pp. 1321-1328.

Cai, D., Li M., Bao, Z., Chen, Z., Wei, W. and Zhang, H. 2010. Study on shadow detection method on high resolution remote sensing image based on HIS space transformation and NDVI index. In Proc. International Conference on Geoinformatics, Beijing, China, pp.1-4.

Dare, P. M. 2005. Shadow analysis in high-resolution satellite imagery of urban areas. Photogrammetric Engineering and Remote Sensing, 71 pp. 169-177.

Ma, H., Qin, Q. and X. Shen. 2008. Shadow segmentation and compensation in high resolution satellite images. In Proc. IEEE IGARSS, Jul. 2008, vol. 2, pp. 1036-1039.

Otsu, N. 1979. A threshold Selection Method from Gray-Level Histograms. IEEE Transactions on Systems, Man and Cybernetics, v. 9, n. 1, pp. 62-69.

Polidorio, A.M., Flores, F.C., Imai, N.N., Tommaselli, A.M. G. and Franco, C., 2003. Automatic shadow segmentation in aerial color images. In: Proc. XVI Brazilian Symposium on Computer Graphics and Image Processing, São Carlos, Brazil, 12-15 October, pp. 270-277. 
Sanin, A., Sanderson, C. and Lovell, B. 2012. Shadow detection: A survey and comparative evaluation of recent methods. Pattern Recognit., 45, pp. 1684-1695.

Soille, P. 2004. Morphological Image Analysis. Berlin. Springer-Verlag. 391p.

Tsai, V.U.D., 2006. A comparative study on shadow compensation of color aerial images in invariant color models. IEEE Transactions on Geoscience and Remote Sensing, 44 (6), pp. 1661-1671.

Wang, Q., Adiku, S., Tenhunen, J. and Granier, A. 2005. On the relationship of NDVI with leaf area index in a deciduous forest site Remote Sensing of Environment, 94, pp. 244-255

Wiedemann, C., Heipke, C., Mayer, H. and Jamet, O., 1998. Empirical evaluation of automatically extracted road axes. In: Empirical Evaluation Methods in Computer Vision. IEEE Computer Society Press, pp. 172-187. 